\title{
Multimedia over Massive MIMO Wireless Systems
}

\author{
Haichao Wang*, Xiaohu Ge*, Ran $\mathrm{Zi}^{*}$, Jing Zhang*, Qiang $\mathrm{Ni}^{\dagger}$ \\ ${ }^{*}$ School of Electronic Information and Communications \\ Huazhong University of Science \& Technology, Wuhan, China \\ ${ }^{\dagger}$ School of Computing and Communications, \\ Lancaster University, Lancaster LA1 4WA, UK. \\ Corresponding author: Xiaohu Ge, email: xhge@ mail.hust.edu.cn
}

\begin{abstract}
To satisfy the massive wireless traffic transmission generated by multimedia applications, the massive multi-inputmulti-output (MIMO) wireless system has emerged as a possible solution for future 5G wireless communication systems. However, the mutual coupling effect of massive MIMO systems has a negative effect potential on the wireless capacity. In this paper, the receive diversity gain is first defined and analyzed for massive MIMO wireless systems. Furthermore, we propose an effective capacity with the mutual coupling effect and the quality of service (QoS) statistical exponent constraint for multimedia massive MIMO wireless systems. Based on numerical results, the effective capacity approaches the effective capacity with the antenna spacing of $d=0.5 \lambda$ when the antenna spacing is increased in the massive MIMO antenna array.
\end{abstract}

Index Terms-Mutual coupling, massive MIMO system, effective capacity, QoS statistical exponent.

\section{INTRODUCTION}

To meet the rapid growth in wireless traffic driven by multimedia applications, the massive multi-input-multi-output (MIMO) technology is presented as one of the key technologies for the fifth generation $(5 \mathrm{G})$ wireless communication systems [1], [2]. Recent studies validate that massive MIMO wireless communication systems have great potential capability to improve the wireless transmission rate in cellular networks [3]. However, considering the limited available physical space for deploying a large number of antenna elements in both base stations (BSs) and mobile terminals, the mutual coupling effect among antenna elements is inevitable for massive MIMO wireless communication systems [4]. On the other hand, the realistic transmission capacity with the quality of service (QoS) constraint is different with the conventional shannon capacity for multimedia wireless systems [5]. Therefore, it is important to evaluate the impact of the mutual coupling effect and the QoS constraint on the multimedia massive MIMO wireless systems.

Effects of the mutual interaction between two or more antennas have been widely studied in the traditional area of antenna propagation and antenna arrays, signal processing [6]-[10]. Based on the theoretical analysis and experimental measurements, the performance of the antenna array was compared with or without the mutual coupling effect [6]. It is shown that mutual coupling significantly affects the performance of adaptive antenna arrays for either large or small inter-element spacing because the steering vector of the antenna array has to be modified both in phase and amplitude to include changes in desired signal vectors [7]. In a reverberation chamber, the impact of mutual coupling on the radiation efficiency, diversity gain, correlation, and channel achievable rate of MIMO systems was measured in [8]. Simulation results reveal that the mutual coupling leads to a substantial decrease in the achievable rate and reduces the degrees of freedom in the wireless channel spatial diversity. The impact of mutual coupling induced by two closed spaced minimum scattering antennas at the subscriber unit on a $2 \times 2$ MIMO channel was investigated in [9]. To restore signals as received by isolated elements in the absence of coupling, a new technique was developed to compensate for mutual coupling in a small antenna array [10]. However, the signal-to-noise ratio (SNR) enhancement is achieved at the cost of a decreased frequency bandwidth and a increased sensitivity to dissipative losses in antennas and matching network structures.

In conventional wireless multimedia communication systems, some studies have been carried out [11]-[13]. To guarantee the QoS in wireless networks, a statistical QoS constraint model was built to analyze the queue characteristics of data transmissions [11]. Based on the effective capacity of the block fading channel model, a QoS driven power and rate adaptation scheme over wireless links was proposed for mobile wireless networks [12]. Furthermore, by integrating information theory with the effective capacity, some QoS-driven power and rate adaptation schemes was proposed for diversity and multiplexing systems [13]. Simulation results showed that multi-channel communication systems can achieve both high throughput and stringent QoS at the same time. However, the investigation of the effective capacity for multimedia massive MIMO wireless systems considering mutual coupling effect and the QoS constraint is surprisingly rare in the open literature.

Motivated by the above gaps, in this paper we consider a multimedia massive MIMO wireless communication system with the mutual coupling effect, and derive the effective capacity considering the QoS statistical exponent constraint. The contributions and novelty of this paper are summarized as follows.

1) The receive diversity gain is defined to evaluate the impact of mutual coupling on the BS rectangular antenna array in the massive MIMO wireless communication systems. 


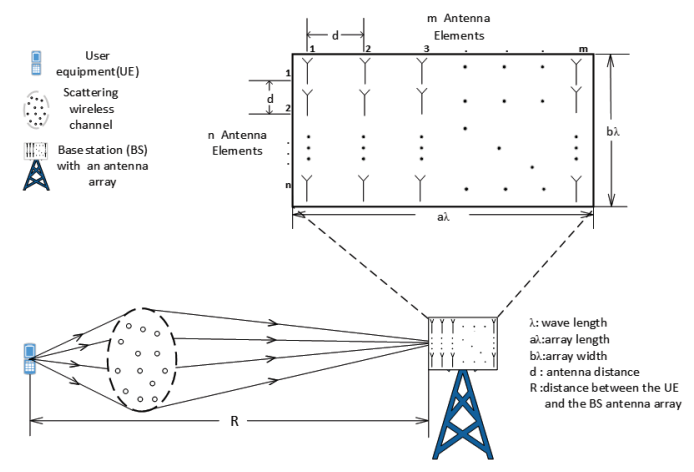

Fig. 1. System model.

2) Based on the mutual coupling effect and the QoS statistical exponent constraint, the effective capacity of multimedia massive MIMO wireless communication systems is proposed and the upper bound of the effective capacity is derived.

3) Based on numerical results, the impact of the mutual coupling and the QoS statistical exponent on the effective capacity is evaluated for multimedia massive MIMO wireless communication systems and some interesting observations are presented.

The remainder of this paper is outlined as follows. Section II describes a system model for massive MIMO wireless communications where the BS has a 2D antenna array. In Section III, the achievable rate of massive MIMO wireless communication systems with mutual coupling is derived. Furthermore, the effective capacity with the QoS statistical exponent is proposed for multimedia massive MIMO wireless systems with the mutual coupling effect. Simulation results and analysis are presented in Section IV. Finally, the conclusion is drawn in Section V.

\section{SYSTEM MODEL}

A massive MIMO wireless communication scenario is illustrated in Fig. 1. In this paper, we focus on the uplink transmission between a BS with a $2 \mathrm{D}$ rectangular antenna array and a single-antenna user equipment (UE).

The wavelength of the wireless transmission wave is denoted as $\lambda$. Moreover, the length and width of the rectangular antenna array are $a \lambda(a \geq 1)$ and $b \lambda(b \geq 1)$, respectively. The antenna spacing of the rectangular antenna array is denoted by $d$. Each row and column of the rectangular antenna array includes $m$ antennas and $n$ antennas, respectively. The total antenna number of the rectangular antenna array is denoted as $M$. As a consequence, the following relationships are listed:

$$
M=m n,
$$

$$
d=\frac{a \lambda}{m-1}=\frac{b \lambda}{n-1} .
$$

The $M \times 1$ uplink signal vector received at a BS integrated with $M$ antennas is expressed as

$$
\mathbf{y}=\sqrt{S N R_{U E}} \mathbf{K A H} \beta^{1 / 2} x+\mathbf{w},
$$

where $S N R_{U E}$ is the signal-to-noise ratio (SNR) at the transmitter, $x$ is the symbol transmitted from the UE, $\mathbf{w} \in \mathbb{C}^{M \times 1}$ is the additive white Gaussian noise (AWGN) over wireless channels, $\mathbf{K}$ is the mutual coupling matrix which will be modeled in the next section and $\mathbf{H} \sim \mathcal{C N}\left(0, \mathbf{I}_{P}\right)$ is the small scale fading matrix, which is expressed as

$$
\mathbf{H}=\left[h_{1}, \ldots, h_{q}, \ldots, h_{P}\right]^{T} \in \mathbb{C}^{P \times 1},
$$

where $P$ is the number of the independent incident directions in angular domain, $\mathbb{C}^{P \times 1}$ denotes a $P \times 1$ vector, $h_{q} \sim$ $\mathcal{C N}(0,1)$ is the complex small scale fading coefficient received from the $q$ th incident direction, which is given by

$$
h_{q}=h_{q}^{r}+j h_{q}^{i},
$$

where $h_{q}^{r}=\operatorname{Re}\left(h_{q}\right)$ and $h_{q}^{i}=\operatorname{Im}\left(h_{q}\right)$ are the real and imaginary parts of $h_{q}$, respectively. Moreover, $h_{q}^{r}$ and $h_{q}^{i}$ are independently and identically distributed (i.i.d.) Gaussian random variables, i.e., $h_{q}^{r}, h_{q}^{i} \sim \mathcal{C N}\left(0, \frac{1}{2}\right)$. $\beta$ is the large scale fading factor, which is assumed constant with respect to the BS antennas since the geometric and shadow fading change slowly over space.

$P$ can be very large if the propagation environment offers rich scatterers. The performance of massive MIMO systems depends critically on the propagation environment and properties of antenna arrays, but ultimately on the number of degrees of freedom offered by the physical channel [14]. More precisely, a finite-dimensional channel model is used in this paper, where the angular domain is divided into $P$ independent incident directions with $P$ being a large but finite number [14], [15]. Each independent incident direction, corresponding to the azimuth $\phi_{q} \in[-\pi / 2, \pi / 2], q=1, \ldots, P$, and the elevation angle $\theta \in[-\pi / 2, \pi / 2]$, is associated with an $M \times 1$ steering vector $\mathbf{a}\left(\phi_{q}, \theta\right) \in \mathbb{C}^{M \times 1}$. For a rectangular antenna array, the steering vectors of all the $P$ independent incident directions together constitute an $M \times P$ steering matrix $\mathbf{A}$ which is given by

$$
\mathbf{A}=\left[\mathbf{a}\left(\phi_{1}, \theta\right), \ldots, \mathbf{a}\left(\phi_{q}, \theta\right), \ldots, \mathbf{a}\left(\phi_{P}, \theta\right)\right] \in \mathbb{C}^{M \times P},
$$

where $\mathbb{C}^{M \times P}$ denotes an $M \times P$ matrix. The $q$ th column of $\mathbf{A}$ corresponds to the steering vector of the $q$ th incident direction. In other words, when the rectangular antenna array receives the signal from the $q$ th incident direction, the steering matrix $\mathbf{A}$ is denoted as $\mathbf{A}^{q} \in \mathbb{C}^{n \times m}$ for every antenna in the rectangular array. Therefore, the following property holds:

$$
\operatorname{vec}\left(\mathbf{A}^{q}\right)=\mathbf{a}\left(\phi_{q}, \theta\right),
$$

where $\operatorname{vec}(\cdot)$ is the operation of matrix vectorization. Without loss of generality, the antenna located at the first row and the first column of the rectangular antenna array is normalized as 
the reference point with the zero phase response. Moreover, amplitude responses of all the antennas are normalized as 1 . $\mathbf{A}_{c e}^{q},(1 \leq c \leq n, 1 \leq e \leq m)$ is an entry of the steering matrix $\mathbf{A}^{q}$, which is expressed as

$\mathbf{A}_{c e}^{q}=\exp \left\{j \frac{2 \pi}{\lambda}\left[(c-1) d \cos \phi_{q} \sin \theta+(e-1) d \sin \phi_{q} \sin \theta\right]\right\}$.

\section{Mutual Coupling Effect Modeling}

\section{A. Mutual Coupling Matrix}

$\mathbf{K} \in \mathbb{C}^{M \times M}$ is the mutual coupling matrix of the rectangular antenna array with $M$ antennas, which is expressed as [9]

$$
\mathbf{K}=Z_{L}\left(Z_{L} \mathbf{I}_{M}+\mathbf{Z}_{M}\right)^{-1},
$$

where $Z_{L}$ is the antenna load impedance and is constant for every antenna, $\mathbf{Z}_{M}$ is the $M \times M$ mutual impedance matrix, $\mathbf{I}_{M}$ is an $M \times M$ unit matrix. Corresponding to the rectangular antenna array in Fig. 1, the mutual impedance matrix $\mathbf{Z}_{M}$ can be constituted by $n \times n$ sub-matrices,, i.e., $\mathbf{Z}_{M}=\left[\mathbf{Z}_{s t}\right]_{n \times n}$. $\mathbf{Z}_{s t}$ is an $m \times m$ mutual impedance sub-matrix, which denotes mutual impedances between the $m$ antennas located at the $s$ th $(s=1, \ldots, n)$ row and the $m$ antennas located at the $t$ th $(t=1, \ldots, n)$ row in the rectangular antenna array. Therefore, $\mathbf{Z}_{s t}$ is expressed as

$$
\mathbf{Z}_{s t}=\left[\begin{array}{cccc}
z_{11}^{s t} & z_{12}^{s t} & \ldots & z_{1 m}^{s t} \\
z_{21}^{s t} & z_{22}^{s t} & \ldots & z_{2 m}^{s t} \\
\vdots & \vdots & \ddots & \vdots \\
z_{m 1}^{s t} & z_{m 2}^{s t} & \ldots & z_{m m}^{s t}
\end{array}\right],
$$

where $z_{u v}^{s t}$ is the mutual impedance between $A n t_{s u}$ and $A n t_{t v}$ antennas. Ant $t_{s u}$ is the antenna located at the sth row and the $u$ th $(s=1, \ldots, m ; u=1, \ldots, m)$ column of the rectangular antenna array, and $A n t_{t v}$ is the antenna located at the $t$ th row and the $v$ th $(t=1, \ldots, m ; v=1, \ldots, m)$ column of the rectangular antenna array. The distance between $A n t_{s u}$ and $A n t_{t v}$ antennas is $d_{u v}^{s t}=d \sqrt{(t-s)^{2}+(v-u)^{2}}$.

A simplified case is studied here, assuming all $M$ antennas in the rectangular antenna array are dipole antennas with the same parameters. Therefore, the mutual impedance $z_{u v}^{s t}$ only depends on the distance among antennas and can be calculated with the electromotive-force (EMF) method according to [21]. When the distance between adjacent antennas in the rectangular antenna array, i.e., the antenna spacing $d$ is assumed to be fixed, the following matrix properties can be obtained:

$$
\begin{gathered}
z_{u v}^{s t}=z_{(u+1),(v+1)}^{s t}, \\
z_{u v}^{s t}=z_{v u}^{s t} .
\end{gathered}
$$

Furthermore, we can also derive similar properties for the matrix $\mathbf{Z}_{s t}$ :

$$
\begin{gathered}
\mathbf{Z}_{s t}=\mathbf{Z}_{(s+1),(t+1)}, \\
\mathbf{Z}_{s t}=\mathbf{Z}_{t s} .
\end{gathered}
$$

With (10) to (14), which is concluded from the properties of the uniform rectangular antenna array, the mutual impedance matrix $\mathbf{Z}_{M}$ can be easily obtained. What needs to be noted here is that equations (10) to (14) greatly reduce the computation complexity comparing with directly calculating the $M \times M$ entries of the mutual impedance matrix, especially when the antenna number $M$ is large.

\section{B. Receive Diversity Gain Models}

For a fixed space of antenna array in the BS, the number of antennas is inversely proportional to the antenna spacing, i.e., the larger the antenna number corresponds the smaller the antenna spacing. The receive diversity gain of the massive MIMO wireless communication system increases with the increase of the antenna number, while it reduces with the decrease of the antenna spacing [16]. Therefore, when the antenna array of the massive MIMO system is integrated into a fixed area, there exists a tradeoff between the antenna number and antenna spacing. Hence, it is significant to evaluate the effect of mutual coupling on the receive diversity gain of the massive MIMO wireless communication system with this tradeoff.

First, we fix the antenna spacing of the rectangular antenna array and the antenna number receive diversity gain $\mathbb{G}_{M}$ is defined as

$$
\mathbb{G}_{M}=\xi_{M}^{d_{\min }}-\xi_{M_{\min }}^{d_{\min }},
$$

where $\xi_{M}^{d_{\min }}$ is the expectation of the receive SNR at the BS with the minimum antenna spacing $d_{\min }$ and the antenna number $M, \xi_{M_{\min }}^{d_{\min }}$ is the expectation of the receive SNR at the BS with the minimum antenna spacing $d_{\min }$ and the minimum antenna number $M_{\min }$.

Second, we fix the antenna number in the massive MIMO system and the antenna spacing receive diversity gain $\mathbb{G}_{d}$ is defined as

$$
\mathbb{G}_{d}=\xi_{M_{\min }}^{d}-\xi_{M_{\min }}^{d_{\min }},
$$

where $\xi_{M_{\min }}^{d}$ is the expectation of the receive SNR at the BS with the minimum antenna number $M_{\min }$ and the antenna spacing $d . \xi_{M_{\min }}^{d_{\min }}$ is the same as that of $\mathbb{G}_{M}$ in (15), which is configured as the baseline for both $\mathbb{G}_{M}$ and $\mathbb{G}_{d}$.

Based on (15) and (16), the total receive diversity gain with the antenna number and spacing is defined as

$$
\mathbb{G}=\mathbb{G}_{M}+\mathbb{G}_{d} .
$$

In order to get the receive diversity gain in (17), we try to derive the expectation of the receive SNR in (15) and (16). The BS is assumed to have perfect channel state information and the maximum-ratio combining (MRC) scheme is assumed to be used for user signal detection. With the antenna number being $M$ and the antenna spacing being $d$, the received signal at the BS passing through the MRC detector is expressed as [17]

$$
\tilde{y}=\mathbf{G}^{\dagger} \mathbf{y}=\sqrt{S N R_{U E}} \mathbf{G}^{\dagger} \mathbf{G} x+\mathbf{G}^{\dagger} \mathbf{w},
$$

where $\mathbf{G}^{\dagger}$ is the conjugate transpose of $\mathbf{G}$ with

$$
\mathbf{G}=\mathbf{K A H} \beta^{1 / 2} .
$$


Furthermore, the SNR at BS is expressed as

$$
S N R_{B S}=\frac{S N R_{U E}\left\|\mathbf{G}^{\dagger} \mathbf{G}\right\|^{2}}{\left\|\mathbf{G}^{\dagger} \mathbf{G}\right\|}=S N R_{U E}\left\|\mathbf{G}^{\dagger} \mathbf{G}\right\| \text {. }
$$

Then the expectation of the SNR with antenna number $M$ and antenna spacing $d$ at the BS is derived by

$$
\begin{aligned}
\xi_{M}^{d} & =\mathbb{E}\left(S N R_{B S}\right) \\
& =S N R_{U E} \mathbb{E}\left(\mathbf{G}^{\dagger} \mathbf{G}\right) \\
& =S N R_{U E} \mathbb{E}\left\{\operatorname{trace}\left(\mathbf{G G}^{\dagger}\right)\right\} \\
& =S N R_{U E} \beta \mathbb{E}\left\{\operatorname{trace}\left(\mathbf{K A H} \mathbf{H}^{\dagger} \mathbf{A}^{\dagger} \mathbf{K}^{\dagger}\right)\right\} \\
& =S N R_{U E} \beta \operatorname{trace}\left\{\mathbf{K A} \mathbb{E}\left(\mathbf{H H}^{\dagger}\right) \mathbf{A}^{\dagger} \mathbf{K}^{\dagger}\right\} \\
& =S N R_{U E} \beta \operatorname{trace}\left(\mathbf{K A A} \mathbf{A}^{\dagger} \mathbf{K}^{\dagger}\right)
\end{aligned}
$$

where $\mathbb{E}(\cdot)$ is the expectation operation. Substituting (20) into (15), (16) and (17), and further replacing $M$ and $d$ with $M_{\min }$ and $d_{\text {min }}$, the receive diversity gains as $\mathbb{G}_{M}, \mathbb{G}_{d}$, and $\mathbb{G}$ are solved.

\section{Effective Capacity with Mutual Coupling Effect}

Based on (20), the available rate, i.e., the shannon capacity within a frame duration for the massive MIMO wireless communication system considering mutual coupling is derived as

$$
\begin{aligned}
R & =\log _{2}\left(1+S N R_{B S}\right) \\
& =\log _{2}\left(1+S N R_{U E} \mathbf{G}^{\dagger} \mathbf{G}\right) \\
& =\log _{2}\left(1+\beta S N R_{U E}\left(\sum_{l=1}^{P}\left|h_{l}\right|^{2} \sum_{m=1}^{P} \mathbf{Q}_{l m}\right)\right)^{\prime}
\end{aligned}
$$

where $Q_{l m},(1 \leqslant l \leqslant P, 1 \leqslant m \leqslant P)$, is the entry of $\mathbf{Q}, \mathbf{Q}=$ $\mathbf{A}^{\dagger} \mathbf{K}^{\dagger} \mathbf{K A}$. Based on results in [22], the effective capacity considering multimedia constraints is defined as

$$
C_{E}(\theta)=-\frac{1}{\theta T} \log \left(\mathbb{E}\left\{e^{-\theta T B R}\right\}\right),
$$

where $\theta$ is the QoS statistical exponent and $B$ denotes the bandwidth for multimedia communication systems. Without loss of generality, the fading process over wireless channels is assumed to be independent of each other and keeps invariant within a frame duration $T$.

Based on the result in (21), (22) can be simplified as

$$
\begin{aligned}
& C_{E}(\theta) \\
& =-\frac{1}{\theta T} \log \left(\mathbb{E}\left\{e^{-\theta T B R}\right\}\right) \\
& =-\frac{1}{\theta T} \log \left(\mathbb { E } \left\{e^{\left.\left.-\theta T B \log _{2}^{\left(1+S N R_{B S}\right)}\right\}\right)}\right.\right. \\
& =-\frac{1}{\theta T} \log \left(\mathbb{E}\left\{\left(e^{\log _{2}^{\left(1+S N R_{B S}\right)}}\right)^{-\theta T B}\right\}\right) \\
& =-\frac{1}{\theta T} \log \left(\mathbb{E}\left\{\left(1+S N R_{B S}\right)^{-\frac{\theta T B}{\log (2)}}\right\}\right)
\end{aligned}
$$

where $S N R_{B S}=\beta S N R_{U E}\left(\sum_{l=1}^{P}\left|h_{l}\right|^{2} \sum_{m=1}^{P} \mathbf{Q}_{l m}\right)$.

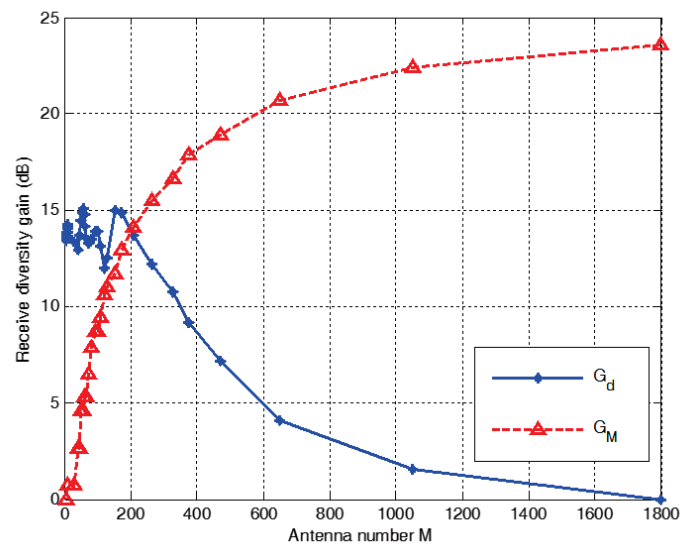

Fig. 2. Receive diversity gain with respect to the antenna number and the antenna distance.

It is obvious that $f(x)=x^{-a},(a>0)$ is a convex function, so the upper bound of the effective capacity can be reached according to Jensen's inequality as follows,

$$
\begin{aligned}
& C_{E}(\theta) \\
& =-\frac{1}{\theta T} \log \left(\mathbb{E}\left\{\left(1+S N R_{B S}\right)^{-\frac{\theta T B}{\log (2)}}\right\}\right) \\
& \leqslant-\frac{1}{\theta T} \log \left(\left\{\mathbb{E}\left(1+S N R_{B S}\right)\right\}^{-\frac{\theta T B}{\log (2)}}\right) \\
& =\frac{B}{\log (2)} \log \left(1+\beta S N R_{U E}\left(\sum_{l=1}^{P} \sum_{m=1}^{P} \mathbf{Q}_{l m}\right)\right)
\end{aligned} .
$$

\section{NUMERICAL RESUlts AND DisCUSSION}

Based on the derived receive diversity gain and the effective capacity, the impact of mutual coupling and the QoS statistical exponent on the performance of multimedia massive MIMO wireless communication systems are investigated in detail. In the following, some default parameters are specified. The ratio of the length and width of the rectangular antenna array is $a / b=2$. The number of the antennas in the rectangular antenna array is 128 [18]. The type of the antenna is the dipole antenna, and the length and diameter of each antenna is $0.5 \lambda$ and $0.001 \lambda$, respectively. Therefore, it is reasonable to set the minimum antenna spacing $d_{\text {min }}$ as $0.2 \lambda$. The load impedance at each antenna is $Z_{L}=50 \mathrm{Ohms}$ [19]. The large scale fading factor is normalized as $\beta=1$ [15], [16], [20]. Here we assume that the BS is surrounded by rich scatters and the incident directions can arrive at arbitrary angles. So the elevation angle $\theta$ and azimuth $\phi_{q}$ follow i.i.d. uniform distributions within $[-\pi / 2, \pi / 2]$. Without loss of generality, the number of independent incident directions is configured as $P=70$. The frame duration $T$ is configured as $1 \mathrm{~ms}$ and the bandwidth $B$ is configured as $1 \mathrm{MHz}$ [22].

In Fig. 2, the antenna number receive diversity gain and the antenna spacing receive diversity gain are investigated. The size of the rectangular antenna array is fixed as $6 \lambda \times 3 \lambda$. 


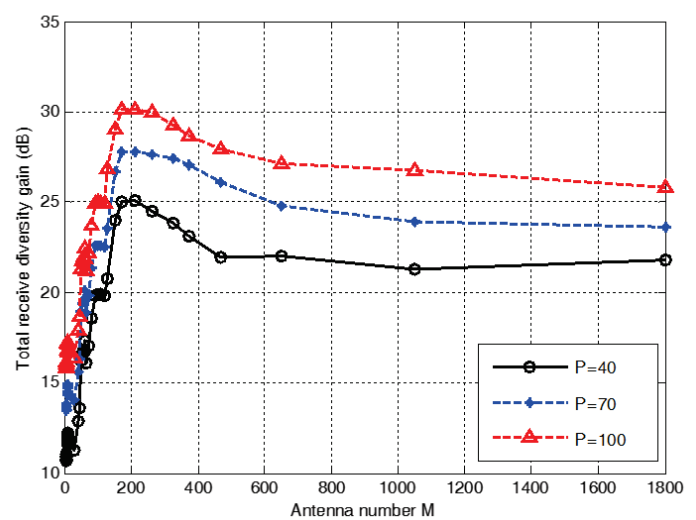

Fig. 3. Total receive diversity gain with respect to the antenna number and the antenna spacing considering different numbers of independent incident directions.

The antenna number receive diversity gain increases with the increase of the antenna number, and the antenna spacing receive diversity gain decreases with the decrease of the antenna distance when the antenna number is larger than 180 . But when the antenna number is less than 180 , the antenna spacing receive diversity gain fluctuates around $14 \mathrm{~dB}$. The reason is that in a fixed-size antenna array, the antenna number being within 0 and 180 corresponds to the antenna spacing being over $0.5 \lambda$. In this range, the mutual impedance fluctuates around a certain value with the increase of the antenna spacing [20]. Therefore, the diversity gain of the antenna spacing fluctuates when the number of antennas is between 0 and 180 .

The total receive diversity gain $\mathbb{G}$ with respect to the antenna number is investigated in the Fig. 3. Here we also fix the size of the antenna array as $6 \lambda \times 3 \lambda$. For a certain number of independent incident directions, the total receive diversity gain firstly increases with the increase of the antenna number when the antenna number is less than 180 , but then starts to decrease although the antenna number keeps growing after the antenna number is larger than or equal to 180 . The range in which the antenna number is less than 180 corresponds to the same range in Fig. 2, where the antenna spacing is larger than $0.5 \lambda$. Then when the antenna is larger than 180 , i.e., the antenna spacing is less than $0.5 \lambda$, the total receive diversity gain declines with the increase of the antenna number. And we can see the maximum of the total receive diversity gain when the antenna number is 180 and the antenna spacing is $0.5 \lambda$. This result can be intuitively explained by the results in Fig. 2, since each curve in Fig. 3 can be viewed as the sum of the two curves with the same number of independent incident directions in Fig. 2. It is also observed from the three curves in Fig. 3 that the larger number of incident direction corresponds to the higher receive diversity gain.

Without loss of generality, the effective capacity is denoted as "EC" and the shannon capacity is denoted as "SC" in Fig. 4. The comparison of the effective capacity with the shannon

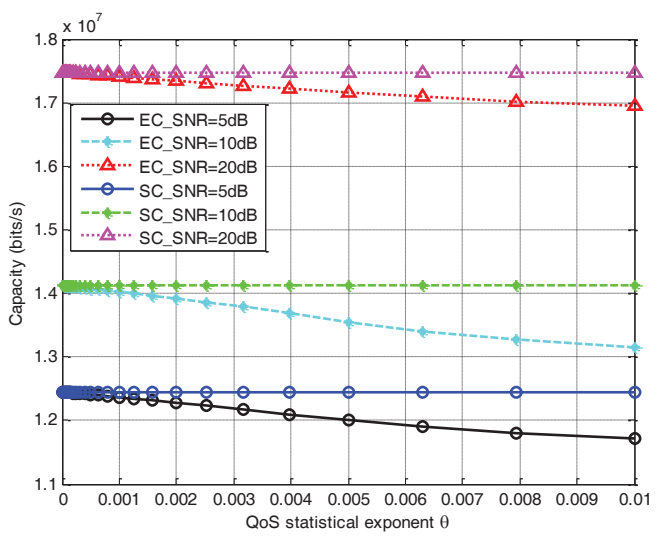

Fig. 4. Comparison of the effective capacity with the shannon capacity.

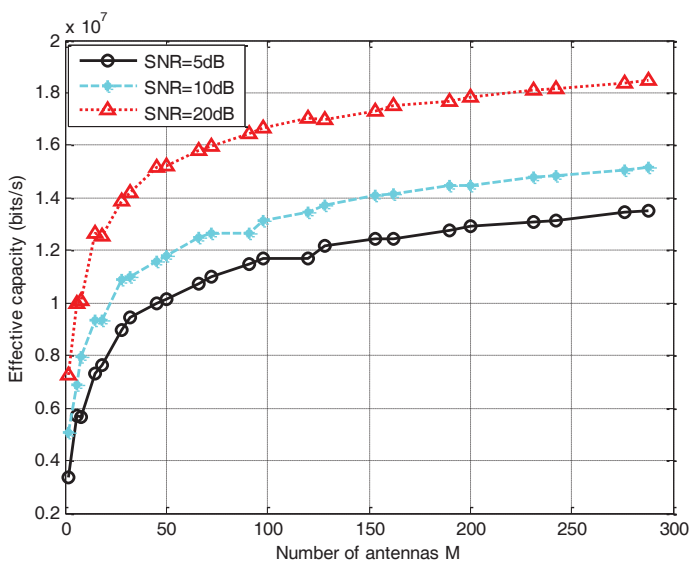

Fig. 5. Effective capacity with respect to the antenna number and the received SNR.

capacity is illustrated in Fig. 4. The size of the antenna spacing is fixed to $0.5 \lambda$ and the antenna number is fixed to 128 . When the QoS statistical exponent is fixed, the effective capacity and the shannon capacity increases with the increase of the SNR over wireless channels. When the SNR is fixed for wireless channels, the effective capacity decreases with the increase of the QoS statistical exponent. Moreover, the effective capacity approximates to the shannon capacity when the QoS statistical exponent $\theta$ approaches 0 .

In Fig. 5, the effective capacity with respect to the antenna number and the received SNR $S N R_{U E}$ is investigated. The antenna spacing is fixed as $0.5 \lambda$. When the SNR is fixed for wireless channels, the effective capacity increases with the increase of the antenna number. When the antenna number is fixed, the effective capacity increases with the SNR value.

Fig. 6 illustrates the effective capacity with respect to the antenna spacing and the QoS statistical exponent. When the antenna spacing is fixed, the effective capacity decreases with the increase of the QoS effective exponent. When the 


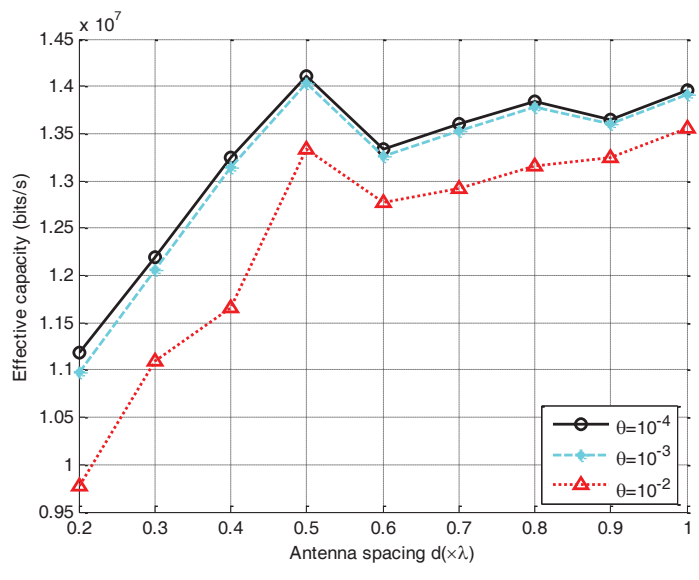

Fig. 6. Effective capacity with respect to the antenna spacing and the QoS statistical exponent $\theta$.

QoS statistical exponent is fixed, the effective capacity first increases with the increase of the antenna spacing. After the antenna spacing is larger than the threshold, i.e., $d=0.5 \lambda$, the effective capacity presents fluctuation and approaches to the effective capacity with the antenna spacing $d=0.5 \lambda$.

\section{CONCLUSION}

In this paper, the impact of mutual coupling on the massive MIMO wireless communication system has been modeled and analyzed. To evaluate the mutual coupling effect, the receive diversity gain is defined and analyzed through numerical results. It is shown that the antenna spacing and antenna number in the rectangular antenna array have an obvious impact on the performance of massive MIMO wireless communication systems considering the mutual coupling effect. Furthermore, the effective capacity with the QoS statistical exponent is derived for multimedia massive MIMO wireless systems considering the mutual coupling effect. Numerical results show that the effective capacity approaches the effective capacity with antenna spacing $d=0.5 \lambda$ when the antenna spacing is increased in the massive MIMO antenna array. For our future work, we will explore a more efficient precoding scheme to improve the multimedia performance of massive MIMO wireless communication systems considering the mutual coupling and QoS statistical exponent constraints.

\section{ACKNOWLEDGMENT}

The authors would like to acknowledge the support from the International Science and Technology Cooperation Program of China under the grants 2014DFA11640 and 0903, the National Natural Science Foundation of China (NSFC) under the grants 61271224, NFSC Major International Joint Research Project under the grant 61210002, the Fundamental Research Funds for the Central Universities under the grants 2013ZZGH009 and 2014QN155. This research is partially supported by EU FP7-PEOPLE-IRSES, project acronym S2EuNet (grant no.
247083), project acronym WiNDOW (grant no. 318992) and project acronym CROWN (grant no. 610524).

\section{REFERENCES}

[1] E. G. Larsson, F. Tufvesson and O. Edfors, "Massive MIMO for next generation wireless systems," IEEE Commun. Mag., vol. 52, no. 2, pp. 186-195, Feb. 2014.

[2] A. Pitarokoilis, S. K. Mohammed and E. G. Larsson, "On the optimality of single-carrier transmission in large-scale antenna systems," IEEE Wireless Commun. Lett., vol. 1, no. 4, pp. 276-279, Aug. 2012.

[3] F. Rusek, D. Persson and B. K. Lau, "Scalling up MIMO: opportunities and challenges with very large arrays," IEEE Signal Proces. Mag., Vol. 30, no. 1, pp. 40-60, Jan. 2013.

[4] Z. Xu, S. Sfar and R. Blum, "Receive antenna selection for closely-spaced antennas with mutual coupling," IEEE Trans. Wireless Commun., vol. 9, no. 2, pp. 652-661, Feb. 2010.

[5] D. Niyato, E. Hossain and K. Dong, "Joint admission control and antenna assignment for multiclass QoS in spatial multiplexing MIMO wireless networks," IEEE Commun. Mag., vol. 8, no. 9, pp. 4855-4865, Sep. 2010.

[6] J. Andersen and H. Rasmussen, "Decoupling and descattering networks for antennas," IEEE Trans. Antennas and Propag., vol. 24, no. 6, pp. 841-846, Nov. 1976

[7] I. J. Gupta and A. A. Ksienski, "Effect of mutual coupling on the performance of adaptive arrays," IEEE Trans. Antennas Propagat., vol. 31, no. 5, pp. 785-791, Sept. 1983.

[8] P. S. Kildal and K. Rosengren, "Correlation and capacity of MIMO systems and mutual coupling, radiation efficiency, and diversity gain of their antennas: simulations and measurements in a reverberation chamber," IEEE Commun. Mag., vol. 42, no. 12, pp. 104-112, Dec. 2004.

[9] B. Clerckx, C. Craeye, D. Vanhoenacker-Janvier and C. Oestges, "Impact of antenna coupling on 2 × 2 MIMO communications," IEEE Trans. Veh. Technol., vol. 56, no. 3, pp. 1009-1018, May 2007.

[10] H. Steyskal and J. S. Herd, "Mutual coupling compensation in small array antennas," IEEE Trans. Antennas and Propag., vol. 38, no. 12, pp. 1971-1975, Dec. 1990.

[11] D. Wu, R. Negi, "Effective capacity: a wireless link model for support of quality of service," IEEE Trans. Wireless Commun., vol. 2, no. 4, pp. 630-643, July 2003.

[12] J. Tang, X. Zhang, "Quality-of-service driven power and rate adaptation over wireless links," IEEE Trans. Wireless Commun., vol. 6, no. 8, pp. 3058-3068, Aug. 2007.

[13] J. Tang, X. Zhang, "Quality-of-service driven power and rate adaptation for multichannel communications over wireless links," IEEE Trans. Wireless Commun., vol. 6, no. 12, pp. 4349-4360, Dec. 2007.

[14] H. Q. Ngo, E. Larsson and T. Marzetta, "The multicell multiuser MIMO uplink with very large antenna arrays and a finite-dimensional channel," IEEE Trans. Commun., vol. 61, no. 6, pp. 2350-2361, June 2013.

[15] H. Q. Ngo, E. G. Larsson, and T. L. Marzetta, "Analysis of the pilot contamination effect in very large multicell multiuser MIMO systems for physical channel models," Proc. IEEE ICASSP'11, pp. 3464-3467, May 2011.

[16] C. Masouros, M. Sellathurai, and T. Ratnarajah, "Large-scale MIMO transmitters in fixed physical spaces: the effect of transmit correlation and mutual coupling," IEEE Trans. Commun., vol. 61, no. 7, pp. 2794 2804, July 2013.

[17] D. N. C. Tse and P. Viswanath, Fundamentals of Wireless Communications. Cambridge Univ. Press, 2005.

[18] J. Hoydis, S. ten Brink and M. Debbah, "Massive MIMO in the UL/DL of cellular networks: how many antennas do we need?" IEEE J. Select. Areas Commun., vol. 31, no. 2, pp. 160-171, Feb. 2013.

[19] S. Shen, M. R. McKay and R. D. Murch, "MIMO systems with mutual coupling: how many antennas to pack into fixed-length arrays?" in Proc. IEEE ISITA'10, pp. 531-536, Oct. 2010.

[20] H. Q. Ngo, E. G. Larsson and T. L. Marzetta, "Massive MU-MIMO downlink TDD systems with linear precoding and downlink pilots," in Allerton Conference on Communication, Control, and Computing, pp. 293-298, Oct. 2013.

[21] C. A. Balanis, Antenna Theory: Analysis and Design. John Wiley and Sons, 2012

[22] X. Ge, X. Huang, Y. Wang, et. al., "Energy efficiency optimization for MIMO-OFDM mobile multimedia communication systems with QoS constraints," IEEE Trans. Veh. Technol. , vol. 63, no. 5, pp. 2127-2138, June 2014. 